\title{
Role of a Protease in Natural Activation of Clostridium botulinum Neurotoxin
}

\author{
BIBHUTI R. DAS GUPTA AND H. SUGIYAMA \\ Food Research Institute and Department of Bacteriology, University of Wisconsin, Madison, Wisconsin 53706
}

Received for publication 8 May 1972

\begin{abstract}
All tested proteolytic Clostridium botulinum type A, B, and F strains and certain non-proteolytic $B$ and $F$ cultures produced a protease having trypsin-like substrate specificity; none of the tested type $\mathrm{E}$ (non-proteolytic) strains produced the enzyme. Progenitor toxin (toxic form whose specific toxicity is increased by treatment with trypsin) was found in culture fluid concentrates of all strains not producing the protease; it was also present in some concentrates that had the enzyme. Activation of highly purified type E progenitor toxin (molecular weight 150,000 ) by essentially pure protease from a proteolytic type $B$ culture was always less than that obtained with trypsin. The product of the type E progenitor toxin-protease reaction increased in toxicity when further treated with trypsin. Results suggest that at least two bonds are cleaved by trypsin during activation of type E progenitor toxin to toxin (form manifesting maximal possible specific toxicity). Natural activation of progenitor toxin of proteolytic strains may also involve cleavage of more than one bond.
\end{abstract}

Clostridium botulinum cultures are classified into types ( $A$ through $F$ ) based on the production of serologically distinct neurotoxins. In the cases of types A, B, E, and F, the toxic molecules are believed to be synthesized as progenitor toxins with relatively low specific toxicity or as nontoxic protoxins which are changed into progenitor toxins $(8)$.

Since progenitor toxins increase in toxicity when treated with trypsin $(1,5,7)$, their activation to the form having maximum potential toxicity (henceforth called toxin) probably involves cleavage of specific bonds. One mechanism for the natural activation of progenitor toxin would be through the action of a suitable enzyme(s) produced by the culture. One such enzyme is a protease with trypsin-like specificity (TLE; reference 4) which activates progenitor toxin obtained from young cultures of the same proteolytic type B strain (3).

The present report considers the possibility of a more general role for TLE in the natural activation of progenitor toxin by testing whether or not TLE production by other cultures is correlated with accumulation of progenitor toxin in growth fluids; it also compares the activation of type E progenitor toxin by TLE and trypsin.

\section{MATERIALS AND METHODS}

Representative strains of $C$. botulinum types $\mathrm{A}$, $B, E$, and F include proteolytic and non-proteolytic cultures of types known to have both physiological forms. Their sources have been presented elsewhere (12).

Cultures were grown in $100-\mathrm{ml}$ volumetric cylinders in the medium used previously (4). After incubation for $144 \mathrm{hr}$ at $30 \mathrm{C}$, the culture was centrifuged and the cell-free fluid was made $70 \%$ saturated with $\left(\mathrm{NH}_{4}\right)_{2} \mathrm{SO}_{4}(47.2 \mathrm{~g} / 100 \mathrm{ml})$. The precipitate, formed during holding for $48 \mathrm{hr}$ at $4 \mathrm{C}$ and collected by centrifugation, was suspended in a volume of buffer $[0.05 \mathrm{M}$ tris(hydroxymethyl)amino-

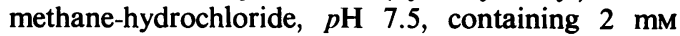
$\mathrm{CaCl}_{2}$ ] equal to $3 \%$ of the starting culture volume. This was dialyzed against the same buffer, clarified by centrifugation, and assayed for TLE and progenitor toxin.

TLE assay was based on amidase activity with $N$ benzoyl-DL-arginyl-p-nitroanilide used as substrate (4). Minor modifications in the assay procedure were incubation of test samples with a reducing agent, dithiothreitol, for $1 \mathrm{hr}$; use of substrate dissolved in

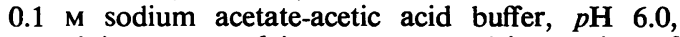
containing $2 \mathrm{~mm}$ calcium acetate; and incubation of reaction mixtures at $30 \mathrm{C}$. The sensitivity limit of the spectrophotometric assay was 0.007 units of enzyme activity per ml of sample.

Toxicity was determined by the intravenous (iv) method in which minutes to death of mice after iv injection is a function of the toxic potency of the sample (2). When desired, death times were converted to $50 \%$ lethal doses (LD L $_{50}$ ) per unit volume by using standard curves which were prepared from iv death times of mice challenged with known intraperitoneal (ip) $\mathrm{LD}_{50}$ amounts of the corresponding toxic form. Death times were means obtained with five mice. 
Progenitor toxin was determined as the toxic form whose specific toxicity could be increased by incubation with trypsin. Trypsinization was by incubating at $37 \mathrm{C}$ for $1 \mathrm{hr}$ a mixture of $1 \mathrm{ml}$ of sample and $0.1 \mathrm{ml}$ of trypsin $(40 \mu \mathrm{g})$, both in $0.01 \mathrm{M}$ $\mathrm{Na}_{2} \mathrm{HPO}_{4}-\mathrm{NaH}_{2} \mathrm{PO}_{4}$ buffer, $p \mathrm{H}$ 6.0. Crystalline trypsin (grade A, lot 72354; Calbiochem, Los Angeles, Calif.) was used routinely. Trypsin, freed of chymotryptic activity by treatment with L-tosylamido-2-phenyl-ethylchloromethyl ketone (Worthington Biochemical Corp., Freehold, N.J.), was used in experiments that compared the effects of trypsin and TLE on type E progenitor toxin. Soy bean trypsin inhibitor was from Worthington Biochemical.

TLE, prepared from type B strain Lamanna (=Okra), was an essentially homogeneous preparation (4). Type E progenitor toxin, isolated from strain Alaska E43, was a preparation obtained in a purification study (R. Heimsch and H. Sugiyama, Bacteriol. Proc., p. 69, 1971) and was a highly purified toxin of molecular weight 150,000 .

\section{RESULTS}

TLE activity demonstrated in culture fluid concentrates of the several $C$. botulinum strains is considered by two criteria to be due to the same enzyme. Like the purified TLE (4), activity in all cases was about one-third less at $p \mathrm{H} 7.5$ than at $p \mathrm{H} 6.0$ and was strictly dependent on preincubation with dithiothreitol.

Table 1 shows that TLE was produced by all cultures which are considered proteolytic on the basis of their ability to digest coagulated egg white and meat particles (11). However, two (type B strain 2129 and F strain 2382), nonproteolytic by this criterion, produced the enzyme. The essentially pure TLE acted as a gelatinase when used to charge wells cut in $12 \%$ gelatin gel made in $0.1 \mathrm{M}, p \mathrm{H} 6.0$, acetate buffer containing $2 \mathrm{mM} \mathrm{Ca}^{2+}$ and kept at $20 \mathrm{C}$ for 6 to $10 \mathrm{hr}$. Since $C$. botulinum strains categorized as non-proteolytic are capable of breaking down gelatin (11), it may be that a strain producing a low level of TLE is gelatinolytic but not overtly ovolytic.

The relationship between the presence of TLE and progenitor toxin in the culture fluid concentrates is shown in Table 1. Progenitor toxin was present in all samples which did not have TLE. The converse relationship, negative progenitor and positive TLE, did not always hold. Of particular interest was culture fluid of type B strain 2131 which possessed a high TLE level (7.0 units $/ \mathrm{ml}$ ) but whose toxicity increased fivefold when trypsinized.

Toxicity of highly purified type $\mathrm{E}$ progenitor toxin was increased 70 -fold by $0.5 \mathrm{hr}$ of incubation with trypsin (Table 2). In comparison, maximum activation with TLE occurred after 1
TABLE 1. Screening of C. botulinum cultures (144 hr of incubation at $30 \mathrm{C}$ ) for a protease of trypsinlike specificity (TLE) and accumulation of progenitor toxin

\begin{tabular}{|c|c|c|c|c|}
\hline \multicolumn{3}{|c|}{ Cultures } & \multicolumn{2}{|c|}{$\begin{array}{l}\text { Culture fluid } \\
\text { concentrates }\end{array}$} \\
\hline Type & Strain & $\underset{\mathrm{y}: \mathrm{si}^{a}}{\operatorname{Prot}}$ & $\operatorname{TLE}^{b}$ & $\underset{\operatorname{toxin}^{c}}{\text { Progenitor }}$ \\
\hline \multirow[t]{3}{*}{ A } & 109 & + & ++ & - \\
\hline & Hall & + & ++ & - \\
\hline & 62 & + & ++ & - \\
\hline \multirow[t]{6}{*}{ B } & 2131 & + & ++ & + \\
\hline & Lamanna & + & ++ & - \\
\hline & 169 & + & + & - \\
\hline & 17 & - & - & ++ \\
\hline & 2 & - & - & ++ \\
\hline & 2129 & - & + & ++ \\
\hline \multirow[t]{3}{*}{$\mathrm{E}$} & VH & - & - & ++ \\
\hline & Iwanai & - & - & ++ \\
\hline & Alaska E43 & - & - & ++ \\
\hline \multirow[t]{6}{*}{$F$} & 4404 & + & + & - \\
\hline & Langeland & + & + & - \\
\hline & 4257 & + & + & - \\
\hline & 202 & - & - & ++ \\
\hline & 83 & - & - & ++ \\
\hline & 2382 & - & + & ++ \\
\hline
\end{tabular}

${ }^{a}$ Proteolytic $(+)$ or non-proteolytic $(-)$ based on digestion of coagulated egg white or meat particles.

$b++, 1.5$ to 7.0 units of enzyme activity $/ \mathrm{ml} ;+$, between 0.2 to 0.6 units $/ \mathrm{ml}$; - , no activity.

$c++$, At least 10 -fold toxicity increase by trypsinization; + , 5-fold; - , progenitor toxin absent (less than 2 min difference in death times of mice injected with trypsinized versus nontrypsinized samples).

$\mathrm{hr}$ of treatment and was about one-tenth that obtained with trypsin. Although not shown, higher TLE to progenitor toxin ratios did not result in higher activation.

The quantitatively different activations of type $E$ progenitor toxin by trypsin and TLE suggested that trypsin might further increase the toxicity of the product formed by TLE. When progenitor toxin was incubated with TLE for 1,2 , or $3 \mathrm{hr}$ and then with trypsin for $1 \mathrm{hr}$, the final toxicity was always two- to threefold higher than that produced by TLE alone (Table 2). Similar results were obtained with one-tenth concentrations of the enzymes. In no case, however, was this final toxicity as high as when progenitor toxin was treated directly with trypsin.

The iv assay of botulinal toxin is convenient but has the complication that size of the toxic 
TABLE 2. Activation of highly purified type E progenitor toxin with trypsin, C. botulinum protease (TLE), and TLE followed by trypsin ${ }^{a}$

\begin{tabular}{|c|c|c|c|c|c|c|c|}
\hline \multicolumn{2}{|r|}{ Activation } & \multicolumn{6}{|c|}{$\mathrm{LD}_{50 / \mathrm{ml}}$ reaction mixture } \\
\hline Test & Enzyme & $0^{b}$ & 0.5 & 1 & 2 & 3 & 4 \\
\hline 1 & Buffer & $7.8 \times 10^{2}$ & & & $7.8 \times 10^{2}$ & & $7.4 \times 10^{2}$ \\
\hline 2 & Trypsin & & $5.4 \times 10^{4}$ & $5.4 \times 10^{4}$ & $4.9 \times 10^{4}$ & & $2.6 \times 10^{4}$ \\
\hline 3 & TLE & & $4.5 \times 10^{3}$ & $5.6 \times 10^{3}$ & $3.7 \times 10^{3}$ & $2.5 \times 10^{3}$ & $1.5 \times 10^{3}$ \\
\hline 4 & TLE + Trypsin & & & $8.0 \times 10^{3}$ & $1.0 \times 10^{4}$ & $5.2 \times 10^{3}$ & \\
\hline
\end{tabular}

${ }^{a}$ In test 4, progenitor toxin was incubated with TLE for 1, 2, or $3 \mathrm{hr}$ and then further treated with trypsin for $1 \mathrm{hr}$. In all tests, incubation was at $37 \mathrm{C}$; buffer was $0.1 \mathrm{~m}$ sodium acetate-acetic acid, $p \mathrm{H} 6.0$, containing $2 \mathrm{mM} \mathrm{CaCl}$; final concentration of progenitor toxin was $1.4 \mu \mathrm{g} / 0.1 \mathrm{ml}$. Ratios (w/w) of progenitor toxin to enzyme(s) in tests 2,3 , and 4 were $1 \div 4,1 \cdot 5-4,5$ (TLE) : $\$$ (trypsin), respectively.

$b$ Hours of incubation.

molecules affects the death times of the injected mice (9). To correct for possible differences in size of the toxic molecules obtained by the different treatments, $\mathbf{L D}_{50}$ values in the above experiments were interpolated from standard curves derived with material that was comparable to the sample being titrated. Nevertheless, a separate experiment, patterned on part of the work summarized in Table 2, was performed for the purpose of confirming that the iv assay used gave valid data.

Samples were progenitor toxin incubated for 1 hr without enzyme (control), progenitor toxin incubated for $1 \mathrm{hr}$ with TLE, and progenitor toxin incubated successively for $1 \mathrm{hr}$ with TLE and then with trypsin. These samples were assayed by both the iv and the conventional ip assay procedures. In the latter procedure serial twofold dilutions were made in $0.2 \%$ gelatin- 0.02 $\mathrm{M}$ citrate buffer, $p \mathrm{H} 6.5$, and $0.5 \mathrm{ml}$ of each appropriate dilution was injected ip into six mice. Deaths during 4 days were used in the Reed and Muench (10) calculation of $\mathrm{LD}_{50} / \mathrm{ml}$. These were $3.2 \times 10^{3}$ for the control, $1.7 \times 10^{4}$ for the TLE-treated sample $(5 \times$ increase in toxicity), and $8.4 \times 10^{4}$ for the sample treated with TLE and trypsin (5X toxicity increase over TLEtreated material). The iv assay values of the same samples were $1.7 \times 10^{3}, 7.2 \times 10^{3}$, and $2.8 \times 10^{4}$ $\mathrm{LD}_{50} / \mathrm{ml}$, respectively, giving fourfold toxicity increases at each of the two steps. Thus, the absolute values obtained by the two assay procedures varied slightly, but both sets of data permitted the same conclusion that type $\mathrm{E}$ progenitor toxin that has been activated with TLE can be further enhanced in toxicity by trypsin. Additionally, the results indicated that the iv assay procedure can be used to demonstrate trends in toxicity changes.

Toxicity of trypsinized type E progenitor toxin was not increased by subsequent treatment with
TLE. The failure was not due to TLE being inactivated by trypsin since the same results were obtained even when trypsin inhibitor of soybean, which does not inhibit TLE (4), was added to the reaction mixture before TLE.

\section{DISCUSSION}

Activation of type $\mathrm{E}$ progenitor toxin by TLE was always less than that obtained with trypsin. This was not because the TLE used was derived from a different culture type; type $B$ progenitor toxin isolated from the same culture as the enzyme was also activated significantly less by TLE than by trypsin (3; DasGupta, Ph.D. thesis, Bryn Mawr College, Bryn Mawr, Pa., 1970). The further toxicity increase when the product of progenitor-TLE reaction was trypsinized indicates that TLE partially activates progenitor toxin and that trypsin has an additional activating effect.

Both enzymes act on peptide bonds formed by the carboxyl groups of only arginyl and lysyl residues, but in contrast to trypsin TLE has preference for arginyl over lysyl residues (4). This difference suggests a possible explanation for the observations.

Complete activation of progenitor toxin may require cleavage of specific arginyl as well as lysyl bonds. If, because of its activity preference, TLE cleaves only the arginyl bond(s), partial activation would result. Trypsin would then act on the critical but still unaffected lysyl linkage(s) to give further increase in toxicity. Direct treatment with trypsin would not result in incompletely activated progenitor toxin because both arginyl and lysyl bonds would be cleaved. TLE added to this reaction product would not be expected to have further activation effects.

A related possible explanation is that more than one of the same kind of bonds (either arginyl or lysyl) must be broken for maximal 
activation. If this is so, trypsin must act equally well on all the critical linkages whereas TLE cannot.

The interpretations agree with the observations that (i) progenitor toxin is always present when TLE is not produced and (ii) TLE production does not preclude the presence of progenitor toxin, as illustrated by type B strain 2131 . These observations suggest the importance of TLE in the natural activation of progenitor toxin and, also, the need of a different enzyme(s) for complete activation.

The sequence in which the bonds are cleaved may determine the toxicity attained. This possibility arises from type $\mathrm{E}$ progenitor toxin treated sequentially with TLE and trypsin having a lower toxicity than that obtained by treatment with trypsin only. The bond cleaved by TLE may be different from the one affected first by trypsin.

The model proposed for the natural activation of $C$. botulinum progenitor toxin to toxin is the cleavage of at least two bonds by different enzymes. Although the molecule synthesized may not be a nontoxic protoxin but a progenitor toxin having some toxicity, this hypothesis has similarities to the activation of chymotrypsinogen to $\alpha$-chymotrypsin A. In the latter instance, four linkages are broken through the actions of two different enzymes (6).

\section{ACKNOWLEDGMENTS}

Research was supported by the College of Agricultural and Life Sciences, University of Wisconsin, Madison, by Public Health Service grant 5-RO1-FD-00090 from the Food and Drug
Administration, and by contributions from various food companies.

\section{LITERATURE CITED}

1. Bonventre, P. F., and L. L. Kempe. 1960. Physiology of toxin production by Clostridium botulinum types $\mathrm{A}$ and B. IV. Activation of the toxin. J. Bacteriol. 79:24-32.

2. Boroff, D. A., and U. Fleck. 1966. Statistical analysis of a rapid in vivo method for the titration of the toxin of Clostridium botulinum. J. Bacteriol. 92:1580-1581.

3. DasGupta, B. R. 1971. Activation of Clostridium botulinum type B toxin by an endogenous enzyme. J. Bacteriol. 108: 1051-1057.

4. DasGupta, B. R., and H. Sugiyama. 1972. Isolation and characterization of a protease from C. botulinum type B. Biochim. Biophys. Acta 268:719-729.

5. Duff, J. T., G. G. Wright, and A. Yarinsky. 1956. Activation of Clostridium botulinum type E by trypsin. J. Bacteriol. 72:455-460

6. Hartley, B. S. 1964. The structure and activity of chymotrypsin, p. 47-60. In T. W. Goodwin, J. I. Harris,j and B. S. Hartley (ed.), Structure and activity of enzymes. Academic Press Inc., New York.

7. Iida, H. 1970. Activation of C. botulinum toxin by trypsin, p. 336-340. In M. Herzberg (ed.), Proceedings First U.S.Japan Conference on Toxic Microorganisms. U.S. Government Printing Office, Washington, D.C.

8. Lamanna, C., and G. Sakaguchi. 1971. Botulinal toxins and problem of nomenclature of simple toxins. Bacteriol. Rev. 35:242-249.

9. Lamanna, C., L. Spero, and E. J. Schantz. 1970. Dependence of time to death on molecular size of botulinal toxins. Infect. Immunity 1:423-424.

10. Reed, L. J., and H. Muench. 1938. A simple method of estimating fifty per cent end points. Amer. J. Hyg. 27: 493-497.

11. Smith, L. DS., and L. V. Holdeman. 1968. The pathogenic anaerobic bacteria. Charles C Thomas, Springfield, Ill.

12. Sugiyama, H., and G. J. King. 1972. Isolation and taxonomic significance of bacteriophages for non-proteolytic Clostridium botulinum. J. Gen. Microbiol. 70:517-525. 